\title{
Lamarck et Cuvier en révolution
}

Géologie, religion et politique entre violence et progrès (1789-1830)

Lamarck and Cuvier in Revolution. Geology, Religion and Politics between violence and progress (1789-1830)

\section{Cédric Grimoult}

\section{(2) OpenEdition}

\section{Journals}

Édition électronique

URL : http://journals.openedition.org/aes/2099

DOI : 10.4000/aes.2099

ISSN : 2258-093X

\section{Éditeur}

Laboratoire LISAA

\section{Référence électronique}

Cédric Grimoult, "Lamarck et Cuvier en révolution », Arts et Savoirs [En ligne], 12 | 2019, mis en ligne le 24 février 2020, consulté le 10 décembre 2020. URL : http://journals.openedition.org/aes/2099 ; DOI : https://doi.org/10.4000/aes.2099

Ce document a été généré automatiquement le 10 décembre 2020.

Centre de recherche LISAA (Littératures SAvoirs et Arts) 


\title{
Lamarck et Cuvier en révolution
}

\author{
Géologie, religion et politique entre violence et progrès (1789-1830) \\ Lamarck and Cuvier in Revolution. Geology, Religion and Politics between \\ violence and progress (1789-1830)
}

\section{Cédric Grimoult}

\section{Introduction}

1 À partir des années 1796-1801, un affrontement scientifique d'une grande importance se produit au Muséum national d'histoire naturelle. Cette polémique au sujet de la nature, du rythme et du tempo des changements survenus au cours de l'histoire de la Terre concerne principalement deux naturalistes de premier plan: Cuvier et Lamarck. Né en 1769 , le premier arrive à Paris en 1795 et se fait d'emblée remarquer par des interventions brillantes dans le domaine de l'anatomie comparée. Il entre à l'Institut de France dès l'année suivante, et le 15 germinal an IV (4 avril 1796), y lit un article tiré de ses recherches sur les éléphants vivants et fossiles. Ses résultats sont particulièrement clairs : les espèces fossiles se distinguent nettement des formes actuelles et ne doivent pas être confondues avec elles. Cuvier en profite pour présenter sa conception en faveur des révolutions du globe. À plusieurs reprises dans le passé de la Terre, des catastrophes universelles auraient détruit la faune et la flore, expliquant ainsi la disparition des espèces connues à l'état fossile.

\section{Première escarmouche}

2 En comparant le texte de cet article avec un manuscrit préparatoire (Ms $628 \mathrm{du}$ Muséum national d'histoire naturelle), l'historien Richard Burkhardt a montré que Cuvier prétendait initialement que les spécialistes s'entendaient pour affirmer "qu'aucun coquillage existant à présent dans la mer ne se retrouve parmi les pétrifications abondantes dont les continents sont remplis $»^{1}$. Son article traitant de gros animaux, il souhaitait généraliser son propos à l'ensemble des animaux 
préhistoriques. Cependant, après l'intervention probable de Lamarck, professeur des «insectes et des vers" du Muséum, la version publiée évoque seulement des faunes terrestres qui diffèrent de la nôtre ${ }^{2}$. Les naturalistes savaient en effet que les coquillages présentaient un certain nombre de formes "analogues", un adjectif à l'emploi particulièrement flou, mais qui renvoyait à l'identité ou à la très grande ressemblance morphologique entre des formes fossiles et des êtres connus dans la faune actuelle. Bruguière, spécialiste des mollusques et ami de Lamarck, insiste ainsi sur l'intérêt que présentent ces analogues. La Métherie évoque aussi les analogues que lui a montrés Lamarck dans sa collection. Faujas de Saint-Fond, professeur de géologie au Muséum, relève méticuleusement les analogues décrits par Lamarck, afin précisément de tester l'hypothèse catastrophiste de Cuvier.

Né en 1744, Lamarck est un naturaliste très différent de Cuvier. Il s'est surtout fait connaître en tant que botaniste, en publiant, dès 1778, une Flore française, qui reçut l'appui de Buffon, pendant ses dernières années en tant qu'intendant du Jardin du Roi. Collectionneur de coquilles, Lamarck commence à s'y intéresser de près depuis qu'il est nommé professeur "des insectes et des vers", c'est-à-dire de tous les animaux à l'exception des vertébrés, au Muséum, en 1793. Grâce à son ami Bruguière, qui mourut en 1798 pendant son retour d'un voyage d'exploration et de collecte de spécimens au Levant, Lamarck dispose de nombreux spécimens et de guides fiables en matière d'analyse et de comparaison des mollusques, vivants et fossiles. Jusqu'à la lecture par Cuvier de son article au sujet des espèces d'éléphants, Lamarck n'avait pas théorisé au sujet de la zoologie, se contentant de publier de courtes notices descriptives. Nul doute que les prétentions révolutionnaires de Cuvier attirèrent alors son attention et provoquèrent sa réaction spectaculaire.

4 Les préoccupations de Lamarck étaient jusqu'alors concentrées sur la chimie, à laquelle il consacre plusieurs gros volumes théoriques, publiés entre 1794 et 1797. Adepte de l'antique doctrine des quatre éléments (air, eau, terre, feu), Lamarck s'oppose à la nouvelle chimie présentée par Lavoisier et ses disciples, et particulièrement bien représentée à l'Institut de France, avec l'appui de personnalités issues également des champs des mathématiques et de la physique. Le célèbre Laplace ne manque ainsi jamais une occasion de rappeler à Lamarck que ses considérations restent purement théoriques, tandis que les adeptes de la nouvelle chimie appuient leurs découvertes sur des preuves combinant de multiples expériences avec les résultats de leurs calculs. En prônant le recours à la seule observation ${ }^{3}$, Lamarck se révèle isolé par rapport à une communauté scientifique adoptant le style "sévère». En tant que "naturaliste philosophe ", il dédaigne le style austère défendu justement par les nouveaux chimistes. Cette rigueur méthodologique ne correspond pas seulement à la "science sérieuse » visant à se distinguer des spectacles scientifiques à la mode dans le Paris de l'époque, mais surtout à la "science sévère ", qui impose le recours à des mesures de précision, à une nomenclature rigoureuse et à des vérifications expérimentales nombreuses ${ }^{4}$. Tandis que les Lumières désignent la science sous l'appellation de "philosophie naturelle", les réformateurs de la fin du XviII siècle veulent être reconnus comme des spécialistes, qui accordent la priorité aux faits par rapport à la spéculation. Or, Lamarck imite le "roman philosophique " de Descartes, qui «après avoir fait semblant de douter, parle d'un ton si affirmatif de ce qu'il n'entend point ", selon Voltaire ${ }^{5}$. 
Or, justement, Cuvier se range du côté de Laplace et des chimistes. Par opportunisme, car sa géologie présente de multiples aspects spéculatifs ${ }^{6}$, il veut se montrer humble et respectueux des bornes restrictives apportées par l'expérimentation et la mathématisation, méthodes reconnues comme fondamentales par la révolution scientifique $\mathrm{du}$ milieu $\mathrm{du} \mathrm{XvII}^{\mathrm{e}}$ siècle. Il est resté célèbre pour sa formule, lancée d'ailleurs à l'encontre de Lamarck: "Nous, nous savons nous borner à décrire $»^{7}$. Il vouait la métaphysique aux gémonies, et prétendait s'en tenir à «la détermination précise des espèces ", démarche qui " peut servir à détruire des systèmes hasardés, et même à nous donner des lumières sur l'histoire si piquante et si obscure des révolutions de ce globe $»^{8}$.

\section{Changement et traditions}

6 L'article de 1796 sur les éléphants fait date, en raison de la précision de l'analyse anatomique de Cuvier. Celle-ci appuie alors une théorie, fondée sur l'irruption de catastrophes brutales ayant marqué l'histoire de la Terre, qui existait déjà avant la Révolution française, et dont le nom même - celui des « révolutions du globe » avait été proposé plusieurs fois. Dès 1746, Johann Gottlob Krüger évoque ainsi trois grandes révolutions successives - deux tremblements de terre et une inondation - capables de supprimer diverses espèces de la surface du globe. Cette hypothèse des révolutions du globe apparaît directement inspirée de la philosophie stoïcienne de l'époque romaine, comme en témoignent les références aux forces destructrices que sont l'eau et le feu ${ }^{9}$, et reste associée aux réflexions sur le Déluge biblique. Le tremblement de terre détruisant Lisbonne, en 1755, qui horrifie l'Europe entière, offre à cette conception d'une série de changements brutaux et destructeurs, de nouveaux soutiens, au point qu'elle devient assez populaire parmi les savants dans les décennies précédant la Révolution française, et trouve de nombreux soutiens avant même que Cuvier ne lui offre un prestige inédit.

7 La société de Teyler, à Haarlem, aux Pays-Bas, lance donc un concours sur ce sujet: «Jusqu'où peut-on conclure, de ce que l'on connaît de la nature des fossiles, de leurs situations, et de ce qu'on sait d'ailleurs relativement aux formes anciennes et nouvelles de la surface du globe, d'après des fondements incontestables, quels changements ou révolutions générales a subies la surface de la Terre, et combien il doit s'être écoulé de siècles depuis lors? ». Le prix est attribué, en 1787, au naturaliste belge François-Xavier de Burtin (1743-1818). Ce dernier prend clairement partie en faveur de l'extinction, en écrivant : «j'ose assurer que les espèces fossiles non perdues sont une vraie rareté ${ }^{10}$. Ce faisant, il affirme que les formes fossiles présentent toujours quelque différence significative avec les espèces vivantes qui leur ressemblent le plus. Ce savant croit aussi reconnaître l'irruption d'une catastrophe globale qui, longtemps avant le Déluge biblique, « doit avoir anéanti toute la génération, qui existait alors, et bouleversé toute la surface de la Terre $»^{11}$.

8 Le très catholique Burtin adopte ainsi la théorie catastrophiste, selon laquelle les révolutions sont plurielles, ont été brutales et ont anéanti l'ensemble des êtres existant alors sur Terre - autrement dit, la théorie pour laquelle Cuvier est passé à la postérité. Celle-ci se retrouve en écho à la fois chez Nicolas-Antoine Boulanger (1722-1759), bien que ce dernier admette la survie d'au-moins quelques individus, Charles Gilbert Morel de Vindé (1759-1842), ainsi que Georges Wolfgang Knorr (1705-1761) et Jean Ernest Emmanuel Walch (1725-1778). Dans une lettre adressée à Giraud-Soulavie, datée du 21 
mai 1780, l'abbé Roux présente aussi une théorie des trois déluges ${ }^{12}$. Aux théologiens, il apparaît donc désormais que l'extinction respecte davantage la lettre de l'Ancien Testament - qui évoque les géants et le Déluge - que la transformation des espèces.

Burtin promeut ainsi un concordisme explicite, c'est-à-dire une position visant à concilier science et foi, d'autant que la Bible fait référence à des destructions soudaines, voulues par Dieu pour châtier les méchants, et brisant l'ordre habituel du monde. Les adeptes du théisme acceptent l'intervention des miracles en dehors du cadre normal des lois naturelles. Dans un autre livre, Burtin explique l'extinction par des catastrophes répétées :

[...] plusieurs phénomènes semblent indiquer que les climats et la mer ont changé de place par une révolution très prompte. Sans cela pourrait-on concevoir tant d'espèces perdues entre les animaux marins, qui n'auraient pas manqué de suivre les eaux dans une retraite si lente ? ${ }^{13}$

Cette citation illustre le poids considérable de la prise en considération de l'extinction dans l'adoption du catastrophisme. Et l'auteur poursuit avec un argument qui allait connaître une portée considérable, après sa reprise par Cuvier :

D'ailleurs le rhinocéros fossile que Mr Pallas a découvert à la surface du sol en Sibérie, et qui était encore en partie couvert de sa peau avec le poil, n'a pu se conserver que par le froid; ce qui ne pouvait se faire avec un changement successif des climats. ${ }^{14}$

11 En 1791, Jean-André De Luc, naturaliste genevois installé en Angleterre, comprend qu'aux changements lents visibles au sein de chaque couche géologique, s'ajoutent des mutations drastiques entre les diverses couches. C'est tout particulièrement vraisemblable dans le cas de discordances, lorsqu'une couche repose sur plusieurs autres qu'elle recoupe de manière sécante. Le passage de l'ère secondaire à l'ère tertiaire, que nous savons coïncider à la grande extinction de masse qui mit fin, notamment, aux dinosaures, pourrait correspondre, selon De Luc (qui n'employait pas ces termes), à un tel événement spectaculaire. Cette révolution, qui a causé de nombreuses extinctions, ne fut cependant pas totale, mais les formes de vie qui en réchappèrent durent s'adapter aux nouvelles conditions du milieu. Ainsi, De Luc se révèle plus nuancé que Burtin et Cuvier, car sa conception de l'extinction n'empêche pas la transformation des lignées survivantes :

$1^{\circ}$. Toutes les espèces d'animaux marins cessèrent d'exister dans les lieux où cette modification s'opéra immédiatement. $2^{\circ}$. Nombre d'espèces de ces animaux furent détruites dans toute l'étendue de la mer. $3^{\circ}$. Les espèces qui furent conservées sur d'autres fonds, éprouvèrent elles-mêmes de grands changements, et se rapprochèrent ainsi par degrés de celles que nous trouvons dans la mer actuelle. ${ }^{15}$

\section{Des révolutions du globe aux mutations animales}

12 Tout au contraire, dans son article de 1796 sur les éléphants, Cuvier oppose un démenti formel au transformisme: "Quelle que puisse être l'influence du climat pour faire varier les animaux, elle ne va sûrement pas aussi loin : et dire qu'elle peut changer toutes les proportions de la charpente osseuse et la contexture intime des dents, ce serait avancer que tous les quadrupèdes peuvent ne dériver que d'une seule espèce ; que les différences qu'ils présentent ne sont que des dégénérations successives : en un mot, ce serait réduire à rien toute l'histoire naturelle, puisque son objet ne consisterait qu'en des formes variables et des types fugaces ${ }^{16}$. Ce sont presque les mêmes mots qu'avait employés, dès 1775, le théologien Jacques Christophe Valmont de Bomare ${ }^{17}$, 
pour évoquer l'enseignement créationniste traditionnel et s'opposer à Buffon, qui remettait en question la chronologie biblique. Mais Cuvier rénove en quelque sorte cette doctrine officielle, en mettant l'accent sur des mécanismes géologiques compatibles avec une chronologie brève. Il est aussi vraisemblable que le nouveau climat politique et culturel, marqué par la Révolution française et le romantisme, ne bénéficient à cette doctrine catastrophiste qui enflamme les passions. Bien que l'utilisation en géologie du concept et du mot même de révolution se révèle assez largement présent avant 1789, sa popularité entre 1790 et 1830 dut vraisemblablement bénéficier des turbulences politiques, non seulement en France, son épicentre, mais dans l'Europe entière, qui suit l'actualité des événements parisiens avec passion et angoisse.

13 C'est peut-être le cas du naturaliste allemand Johann Friedrich Blumenbach (1752-1840) qui inscrivait initialement sa pensée dans le droit fil des idées de Buffon, adoptant son transformisme limité - présenté en termes de dégénération des races à partir d'un prototype spécifique, diverses espèces pouvant ainsi dériver d'un genre unique - et sa théorie des époques successives dans l'histoire de la Terre. En 1790 cependant, Blumenbach change d'avis et postule plusieurs révolutions totales permettant d'expliquer la destruction des espèces disparues:

Après avoir rempli sa mission dans le passé préadamitique de notre planète, cette création organique fut détruite par une catastrophe totale de sa surface, qui dès lors, resta sans doute en friche, jusqu'au jour où elle fut à nouveau en mesure d'accueillir une nouvelle végétation et une nouvelle création animale. ${ }^{18}$

Son histoire de la Terre implique alors non seulement des révolutions globales, mais aussi des Créations entièrement nouvelles. L'auteur appuie cette conjecture sur un exemple, celui de l'inversion de symétrie du murex actuel par rapport au murex fossile, à la fois très ressemblant et irréductiblement différent : "Ce phénomène n'est pas une conséquence de la dégénérescence, mais au contraire un remodelage $»^{19}$. Ce dernier mot, qui évoque le façonnement des formes dans l'argile, fait évidemment référence au récit de la Genèse. Par opposition à l'embryologiste Albrecht von Haller (1798-1777), qui considère que seuls des athées peuvent chercher à démontrer l'impermanence du monde, Blumenbach adopte l'idée d'une Création recommencée, renouvelée au cours du temps. Pour avoir été brutales et violentes, les révolutions du globe n'en sont pas pour autant complètes, totales, absolues. Pourquoi quelques êtres au moins n'auraientils pas pu survivre aux bouleversements géologiques? Qu'est-ce qui explique la radicalité de la position de Cuvier, laquelle rompt à la fois avec une tradition de prudence et d'éclectisme théorique ${ }^{20}$ et se montre particulièrement fragile au plan méthodologiqu ${ }^{21}$ ?

Sans doute Cuvier a-t-il lu par ailleurs le mémoire de l'astronome et mathématicien Pierre-Simon Laplace (1749-1827), qui souscrit au catastrophisme en décrivant les effets de la rencontre hypothétique entre une comète et la Terre. Laplace reprenait ainsi l'explication du Déluge donnée par William Whiston (1667-1752), déjà commentée en 1742 par Maupertuis. Cuvier peut encore se souvenir du mémoire académique de Joseph Jérôme Lefrançois de Lalande (1732-1807), en 1773, qui causa un certain émoi à travers Paris et les railleries de Voltaire ${ }^{22}$ quand il écrit :

L'axe et le mouvement de rotation changés, les mers abandonnant leur ancienne position pour se précipiter vers le nouvel équateur, une grande partie des hommes et des animaux noyés dans ce déluge universel ou détruits par la violente secousse imprimée au globe terrestre, des espèces entières anéanties, tous les monuments de 
l'industrie humaine renversés, tels sont les désastres que le choc d'une comète a dû produire, si sa masse a été comparable à celle de la Terre. On voit alors pourquoi l'océan a recouvert de hautes montagnes, sur lesquelles il a laissé des marques incontestables de son séjour, on voit comment les animaux et les plantes du Midi ont pu exister dans les climats du Nord, où l'on retrouve leurs dépouilles et leurs empreintes ; enfin on explique la nouveauté du monde moral, dont les monuments certains ne remontent pas au-delà de cinq mille ans. ${ }^{23}$

Charles Laurillard (1783-1853), dessinateur et collaborateur de Cuvier, a d'ailleurs cru que des comètes avaient mis fin au règne animal à plusieurs reprises. Cette idée est en fait très répandue, depuis que les physiciens ont attiré l'attention sur ces corps célestes vagabonds. Newton, Maupertuis, Halley, Laplace ont parlé de ces chocs possibles, qui font écho aux récits mythologiques et bibliques. Lord Byron (1788-1824) le traduit dans le style romantique, qui accorde aussi une large place à la conception de l'éternel retour :

Qui sait si, quand une comète approchera notre globe pour le détruire, comme il le fut souvent et le sera de nouveau, les hommes ne détacheront pas les roches de leurs fondations avec des machines à vapeur, et lanceront des montagnes contre la masse flamboyante incandescente, comme les géants sont censés l'avoir fait ? - Et alors nous aurons de nouvelles traditions de Titans, et de guerres avec le Ciel. ${ }^{24}$

Un autre facteur expliquant le succès du catastrophisme provient de l'explication qu'il produit du phénomène de la fossilisation. Autour de 1800, de nombreux naturalistes s'accordent à penser que l'incrustation des fossiles dans certaines roches est due à un phénomène à la fois rapide et violent ${ }^{25}$. L'image la plus fréquemment employée est celle de la vague puissante, qui précipite les êtres sur les roches sédimentaires, où ils imprimeraient leurs corps solidifiés instantanément. Dès le milieu du $\mathrm{xvIII}^{\mathrm{e}}$ siècle, Georges Wolfgang Knorr et Jean Ernest Emmanuel Walch parlent d'une catastrophe ayant " fait passer ensemble [...] dans le règne souterrain ${ }^{26}$ les animaux " enveloppés tous vivants». De ce que les poissons fossiles sont retrouvés courbés, ces auteurs déduisent «que l'accident, qui les a enfoncés dans le limon ou la fange, leur a fait subir une mort violente $»^{27}$. En 1777, Pallas évoque aussi une vague gigantesque ayant formé d'un seul coup les «montagnes tertiaires » de Russie, contenant - sur une épaisseur de 200 mètres - des rhinocéros, éléphants et buffles censés avoir été transportés depuis les Indes et mêlés à des troncs d'arbres fossilisés ${ }^{28}$. Dieudonné Dolomieu (1750-1801) s'en fait l'écho en $1791^{29}$. Il écrit ainsi, par exemple :

Quelques animaux ont pu se soustraire à ces déluges périodiques en se réfugiant sur les plus hautes sommités ; mais toutes les autres productions de la terre ont dû être emportées dans la mer, pour revenir ensuite avec des corps marins s'ensevelir dans les nouvelles couches. ${ }^{30}$

Cuvier évoque des animaux par millions «laissés à sec, ou ensevelis sous des couches nouvelles, ou jetés avec violence à la côte ${ }^{31}$. La formation des fossiles resterait donc exceptionnelle, propre aux seuls temps des catastrophes :

[...] nous n'avons aucune preuve que la mer puisse aujourd'hui incruster ces coquilles d'une pâte aussi compacte que les marbres, que les grès, ni même que le calcaire grossier dont nous voyons celles de nos couches enveloppées. Encore moins trouvons-nous qu'elle précipite nulle part de ces couches plus solides, plus siliceuses qui ont précédé la formation des bancs coquilliers. Enfin toutes ces causes réunies ne relèveraient pas une seule couche, ne produiraient pas le moindre monticule, ne changeraient pas d'une quantité appréciable le niveau de la mer. ${ }^{32}$ 
Selon Cuvier, c'est en effet la même cause qui doit avoir bouleversé les couches sédimentaires des époques précédentes et causé la disparition des formes de vie contemporaines.

Une telle conception n'est pas partagée par tous les auteurs, loin de là. Reprenant un argument développé par Lavoisier, le célèbre chimiste, dès 1789, Lamarck écrit ainsi :

Je pourrais encore demander comment, dans la supposition d'une catastrophe universelle, auraient pu se conserver une infinité de coquilles délicates que le moindre choc pouvait briser, et dont cependant on trouve maintenant un grand nombre dans leur intégrité parmi les autres fossiles. ${ }^{33}$

Après Benoît de Maillet, il insiste aussi sur l'immense durée qu'a nécessitée la production des couches de craie de Normandie, par exemple, constituée à partir des corps calcaires de minuscules invertébrés ${ }^{34}$. Les fossiles que ces couches renferment témoignent donc du fait que les strates ne représentent nullement la vie sur Terre à un instant donné, mais une collection de formes ayant persisté pendant des millions d'années. Cette interprétation fut définitivement abandonnée dans la seconde moitié du XIX $^{e}$ siècle $^{35}$ mais, vers 1800 , les fossiles semblent encore accréditer l'idée de catastrophe, que Cuvier lie à celle d'extinction.

\section{Lamarck s'oppose frontalement à Cuvier}

22 Lamarck présente un point de vue symétrique à celui de Cuvier, tout aussi excessif que le précédent : il rejette le catastrophisme, l'extinction et la fixité des espèces. Et, au fond, ce qui déplaît sans doute viscéralement dans la conception de son jeune adversaire, c'est sa philosophie spiritualiste et chrétienne sous-jacente. Ami de Rousseau, avec lequel il herborisait autrefois, républicain de cœur, Lamarck fait partie des rares aristocrates demeurés à Paris sous la Terreur, sans se cacher ni connaître apparemment de souci et bénéficie des mutations institutionnelles opérées au Muséum national d'histoire naturelle, ci-devant Jardin du Roi. Pour cet ancien élève dans un lycée jésuite, la déchristianisation permise dans les années 1790 libère la parole des auteurs matérialistes et lui permet de penser les phénomènes physiques uniquement en termes concrets, sans recours au miracle, mais en rapportant systématiquement les faits aux seules lois de la nature. Il insiste ainsi à plusieurs reprises sur la constance des lois physiques et sur la supériorité épistémologique de l'approche naturaliste, qui évite de supposer indûment l'existence d'exceptions miraculeuses ou d'une finalité immanente $^{36}$.

Lamarck est loin de nier que des événements géologiques violents se soient produits dans l'histoire de la Terre. Mais de sévères limitations doivent borner l'imagination des géologues. D'abord, avec un grande modernité, Lamarck refuse d'envisager d'autres types de phénomènes que ceux que l'on peut constater au sein de la nature actuelle. C'est le principe désormais appelé " actualisme » et qui constitue l'un des fondements méthodologiques de la recherche scientifique dans les sciences historiques. C'est en tout cas ce qu'il écrit en 1809: "jamais nous ne devons faire intervenir, dans nos raisonnements, la considération d'objets hors de la nature et sur lesquels il nous sera toujours impossible de savoir quelque chose de positif $\aleph^{37}$. Le catastrophisme n'est pas une hypothèse scientifique, car il ressemble trop à un deus ex machina, au Déluge des géologues biblistes. Cette conjecture invérifiable explique tout sans être compréhensible ${ }^{38}$, comme l'avait déjà dit Buffon. Ensuite, Lamarck insiste sur les preuves de la très grande antiquité du globe, et se risque même à quelques estimations 
chronologiques. Il adopte ainsi le concept et l'expression de "révolutions lentes ", déjà mis en avant par son ami Bruguière ${ }^{39}$. La révolution est le concept le plus éminemment central de la science moderne. Copernic et Galilée ont montré que la Terre tourne sur elle-même et autour du Soleil. Mais la Terre tourne silencieusement, discrètement, de même que, selon Lamarck, ses reliefs et ses habitants se transforment tout en ayant l'air d'être toujours les mêmes. Le renversement de situation est complet - ce qu'implique le mot révolution - mais sans violence. Dans son Hydrogéologie, Lamarck évoque une «lenteur presque inappréciable $»^{40}$. Les changements se produisent " avec un espace de temps d'une durée en quelque sorte immense ${ }^{41}$.

Afin d'invalider la doctrine de Cuvier, il suffit enfin à Lamarck de présenter quelques fossiles analogues appartenant à des couches géologiques séparées par une prétendue révolution du globe. Il renverse alors le raisonnement de son adversaire :

Quoique beaucoup de coquilles fossiles soient différentes de toutes les coquilles marines connues, cela ne prouve nullement que les espèces de ces coquilles soient anéanties, mais seulement que ces espèces ont changé à la suite des temps, et qu'actuellement elles ont des formes différentes de celles qu'avaient les individus dont nous retrouvons les dépouilles fossiles. ${ }^{42}$

Et c'est ainsi en se demandant pourquoi ces espèces ont disparu tandis qu'elles ne sont pas éteintes, qu'il considère d'abord que nombre d'entre elles survivent encore dans des lieux inaccessibles, puis que certaines ont dû se transformer au fil du temps ${ }^{43}$.

Les arguments les plus forts de Lamarck concernent les fossiles les plus récents, qui sont les plus ressemblants des formes vivantes. Dès 1803, il écrit ainsi à propos du Murex tripterus : « son analogue vit actuellement dans la mer des Indes orientales, dans le voisinage de Batavia. Je possède dans mon cabinet, et des individus fossiles de Grignon, et des individus frais qui proviennent de la mer des Indes $»^{44}$. Son transformisme lui permet aussi d'expliquer pourquoi les fossiles les plus proches des êtres vivants sont les plus récents ${ }^{45}$, tandis que Cuvier se borne à observer ce fait: "plus les couches dans lesquelles on trouve ces os sont anciennes, plus ils sont différents de ceux des animaux que nous connaissons aujourd'hui $»^{46}$. Ce dernier considère d'ailleurs qu'il s'agit là du " résultat le plus remarquable et le plus étonnant » qu'il a obtenu dans ses recherches, tout en s'avérant incapable de l'expliquer.

Il reste à comprendre pourquoi Lamarck oppose les deux voies de l'alternative extinction-évolution, alors même que ses collègues s'y refusent, dans la grande majorité des $\mathrm{cas}^{47}$. Une hypothèse logique serait sa crainte de se voir opposer un argument auquel son déterminisme ne pouvait trouver de réponse: si les espèces s'adaptent pour survivre, pourquoi certaines d'entre elles devraient-elles s'éteindre? Vingt ans plus tard, Lyell considère en effet que l'évolution devrait permettre d'éviter l'extinction : celle-ci étant alors conçue comme la preuve du manque de plasticité de l'espèce, et, par conséquent, de sa fixitée ${ }^{48}$. On suppose aujourd'hui que certains changements environnementaux furent trop brutaux pour que les espèces qui les subirent aient pu s'adapter, ou que la réduction lente de la population ait condamné les formes en question à des impasses évolutives. Mais, pour Lamarck, une telle concession reviendrait à retomber dans une alternative soulignée par Cuvier :

Pourquoi les entrailles de la Terre n'ont-elles point conservé les monuments d'une généalogie si curieuse, si ce n'est parce que les espèces d'autrefois étaient aussi constantes que les nôtres, ou du moins parce que la catastrophe qui les a détruites ne leur a pas laissé le temps de se livrer à leurs variations $?^{49}$ 
l'évolution conçue - autrement que de nos jours - comme une tendance au progrès ${ }^{50}$.

\section{Paradoxe révolutionnaire}

Deux autres raison de l'adhésion de Lamarck au transformisme - question majeure dans l'histoire de l'évolutionnisme - peuvent alors être indiquées, bien qu'elles n'aient jamais été envisagées par les spécialistes. La première tient au mécanisme envisagé par Lamarck pour expliquer les transformations du vivant, et principalement son idée selon laquelle «la fonction crée l'organe ». L'expression n'est pas de Lamarck, qui emploie diverses circonlocutions, mais tout à fait conforme à sa pensée. Il serait peutêtre plus juste de dire « le besoin crée l'organe », car Lamarck écrit ainsi, en opposition à l'idée commune : » ce sont, au contraire, les besoins et les usages des parties qui ont développé ces mêmes parties, qui les ont même fait naître lorsqu'elles n'existaient pas et qui, conséquemment, ont donné lieu à l'état où nous les observons dans chaque animal $»^{51}$. C'est en effet en première position, lors de sa toute première prise de position publique en faveur du transformisme, dans son célèbre discours de l'an VIII, que Lamarck présente ce mécanisme des transformations organiques à l'appui de son idée maîtresse ${ }^{52}$. Et force est de reconnaître qu'il n'était pas le seul, à l'époque, pour qui le changement de mode de vie pouvait entraîner, chez les animaux, des transformations notables pouvant conduire à une conception évolutionniste. Outre Buffon, on peut renvoyer à Diderot ${ }^{53}$, Charles-Georges Leroy ${ }^{54}$ et Georges Cabanis ${ }^{55}$.

Tout en semblant puérile de prime abord, la seconde cause possible de l'adhésion de Lamarck que l'on peut ajouter à celles qui sont déjà connues, concerne son opposition systématique à Cuvier. Cette hypothèse s'avère conforme à la théorie synergique de l'évolution des idées, qui tend à faire diverger les hypothèses rivales, de même que des espèces en compétition sont conduites par la sélection naturelle à se différencier. Radicales, les options de Cuvier et de Lamarck sont plus clairement identifiées par les autres membres de la communauté scientifique, et sont passées à la postérité en raison de - plutôt que malgré - leur simplisme et de leur caractère polémique ${ }^{56}$.

31 Pourtant, si Lamarck et Cuvier se rejoignent, c'est dans l'exploitation du modèle révolutionnaire pour penser le changement. Si Lamarck rejette les transformations brutales hors de la géologie, il les accueille favorablement pour ce qui concerne les changements du vivant. Sa biologie - terme qu'il est l'un des tout premiers à utiliser en 1802 - lui fournit deux arguments majeurs en faveur des transformations organiques brutales. Comme chez Benoît de Maillet, la métamorphose du têtard en grenouille révèle, selon Lamarck, comment se produit une révolution organique: l'individu respire d'abord par des branchies, puis par des poumons ${ }^{57}$.

Le second argument majeur de Lamarck ne concerne pas les fossiles, comme on aurait pu s'y attendre, mais la classification animale, qui forme, selon lui, une "échelle régulièrement graduée ", pourvu que l'on considère seulement les groupes supérieurs de la classification ${ }^{58}$. Les espèces, quant à elles, présentent souvent des " ramifications latérales », étant donné que leurs «organes non essentiels» sont soumis aux « influences des circonstances extérieures ». Les historiens n'ont pas encore clairement indiqué à quel point cette conception s'avère contradictoire, dans la mesure où Lamarck lui-même renonce finalement à ranger les espèces, puis les ordres dans une 
série unique, pour proposer finalement un véritable arbre évolutif - ce qui est tout à son honneur - mais contradictoire avec son idée d'une « série unique ${ }^{59}$.

\section{Cuvier radicalise son discours} discours. Il reconnait en effet avoir identifié 49 espèces nouvelles, contre 11 ou 12 identiques aux formes actuelles, et entre 16 et 18 cas indécidables ${ }^{60}$. En 1806, Cuvier révise la position du mastodonte au sein de la classification. Alors qu'il y voyait jusqu'à présent une espèce particulière au sein du genre des éléphants, il en fait désormais un genre nouveau. Autrement dit, les différences morphologiques mineures entre divers fossiles permettent d'établir des espèces distinctes, qui doivent néanmoins être séparées du groupe formé par celles du genre éléphant. C'est le statut de la différence entre éléphants d'Afrique et d'Asie, distinguées par Cuvier, qui fixe le rang d'espèces attribué aux divers mastodontes.

Dans son célèbre Discours préliminaire aux Recherches sur les ossements fossiles de quadrupèdes publié en 1812, Cuvier livre enfin sa conception d'ensemble au sujet de l'histoire de la Terre, marquée par des "révolutions du globe». Il s'y oppose explicitement à l'idée des "révolutions lentes" évoquées par Lamarck ${ }^{61}$. Des révolutions ont lieu, c'est certain. La seule chose qui soit en question, ce sont leurs modalités: lentes, ou brutales, et il faut donc en passer par une estimation chronologique des durées concernées. :

Des êtres vivants sans nombre ont été les victimes de ces catastrophes, les uns ont été détruits par des déluges, les autres ont été mis à sec dans le fond des mers subitement relevé : leurs races même ont fini pour jamais, et ne laissent dans le monde que quelques débris à peine reconnaissables pour le naturaliste. ${ }^{62}$

Le mot « race » insiste sur la généalogie, alors que celui d'espèce renvoie davantage au type morphologique: Cuvier précise donc ici qu'il entend bien évoquer l'élimination définitive de certaines lignées. Dans certains passages, il estime que les révolutions ont été universelles et globales, mettant fin à toute vie. À cette occasion, il reprend l'expression de Lamarck, mais en l'inversant. Ce dernier avait évoqué, en faveur du transformisme, une « véritable chaîne » entre les êtres vivants ${ }^{63}$, « le véritable fil qui lie toutes les productions de la nature $»^{64}$. Au sujet des révolutions du globe, Cuvier écrit : "le fil des opérations est rompu ; la marche de la nature est changée "

Parmi les preuves en faveur du caractère subit de ces révolutions, Cuvier commence, assez curieusement, par l'état de conservation des mammouths et rhinocéros sibériens qui, selon lui «détruisent pour toujours toute idée de révolution lente ${ }^{66}$. Le gel responsable de cette conservation a saisi ces corps dès la mort des animaux et Cuvier croit pouvoir en déduire que «cette gelée éternelle n'a pu s'emparer des lieux où ces animaux vivaient que par la même cause qui les a détruits : cette cause a donc été subite comme son effet $\aleph^{67}$. Dès 1822, John Fleming (1785-1857) observe au contraire que les mammouths et rhinocéros sibériens possédaient une fourrure indiquant leur adaptation au froid, à la différence des espèces actuelles, à la peau glabre ${ }^{68}$. Les autres arguments de Cuvier, tout aussi discutables, proviennent de l'inclinaison des couches sédimentaires ainsi que « des amas de débris et de cailloux roulés, placés en plusieurs endroits entre les couches solides", censés indiquer la violence des événements. L'historien de la géologie Gabriel Gohau écrit à ce propos : « On pourra aussi s'étonner des "révolutions" subites et drastiques qui coupent l'histoire de la Terre, et dont 
Cuvier, si exigeant en d'autres occasions, donne des preuves peu convaincantes $"$ " Force reste donc de constater le caractère extrémiste et simplificateur de cette théorie de la Terre.

Cuvier attaque aussi Lamarck en ce qu'il n'a pu établir directement les filiations supposées entre les formes fossiles et actuelles: «si les espèces ont changé par degrés, on devrait trouver des traces de ces modifications graduelles", tandis que "jusqu'à présent cela n'est point arrivé $\aleph^{70}$. Il ridiculise aussi le mécanisme supposé de ces transformations, ainsi que les exemples peu judicieux mis en avant par Lamarck, comme celui de la girafe.

Les dimensions de cet article ne permettent pas de comparer les productions de Lamarck à celles de plusieurs autres collègues, comme Faujas de Saint-Fond, et surtout Delamétherie, qui ont aussi affronté Cuvier, de manière à la fois plus précoce et souvent pertinente ${ }^{71}$. Il faudrait aussi évoquer l'échec ou le succès de ces conceptions au sein de la communauté scientifique. Elles offrent néanmoins une fenêtre sur l'interférence entre les établissements factuels, les oppositions théoriques, le contexte culturel, la conjoncture politique et les opportunités institutionnelles. En pensant le temps profond, les naturalistes des Lumières ont mis au jour les principales apories qui continuent de diviser les spécialistes aujourd'hui, en particulier pour ce qui concerne les modalités graduelles ou brutales des changements évolutifs ${ }^{72}$.

\section{NOTES}

1. Richard W. Burkhardt Jr, The Spirit of system: Lamarck and evolutionary biology, Harvard, Harvard University Press, 1977, p. 129.

2. Martin J. S. Rudwick, Bursting the limits of time. The reconstruction of geohistory in the Age of Revolution, Chicago et Londres, The University of Chicago Press, 2005, p. 364.

3. Lamarck, Recherches sur les causes des principaux faits physiques, Paris, Maradan, an II (1793-1794), t. II, p. 307-308.

4. Jean-Luc Chappey, «Enjeux sociaux et politiques de la "vulgarisation scientifique" en révolution (1780-1810) », Annales historiques de la révolution française, 2004, n 338, p. 11-51 ; Cédric Grimoult, Science \& société au XVIII siècle en France et en Grande-Bretagne de 1687 à 1789, Paris, Ellipses, 2016, p. 291-292).

5. Voltaire, Le philosophe ignorant, s.l., 1766, p. 6.

6. Cédric Grimoult, Évolutionnisme et fixisme en France: histoire d'un débat. 1800-1882, Paris, CNRS éditions, 1998, p. 74-77.

7. Cité par Robert Courrier, Notice sur Georges Cuvier. Certains aspects de sa carrière, 1970-1971, Archives de l'Académie des Sciences, dossier Cuvier.

8. Cuvier, "Mémoire sur les espèces d'éléphants vivantes et fossiles", Mémoires de l'Institut national des sciences et des arts, fructidor an VII, 1799, t. II, p. 4.

9. Johann Gottlob Krüger, Histoire des anciennes révolutions du globe terrestre, trad.: Deslandes, Amsterdam, 1752, p. 27.

10. François-Xavier Burtin, «Réponse à la question physique proposée par la société de Teyler sur les Révolutions générales qu'a subies la surface de la Terre, et sur l'ancienneté de notre 
globe ", Verhandelinger, uitgegeeven door Teyler's tweede genootschap, Haarlem, John Enschedé en Zoonen et Jan Van Walré, 1789, p. 7.

11. Ibid., p. 224.

12. Jean-Louis Giraud-Soulavie, Histoire naturelle de la France méridionale, Nîmes, Belle, 1782, t. VI, p. 304-391 et notamment p. 318-319.

13. François-Xavier Burtin, Oryctographie de Bruxelles, ou description des fossiles tant naturels qu'accidentels découverts jusqu'à ce jour dans les environs de cette ville, Bruxelles, Impr. de le Maire, 1784 , p. 134, en note.

14. Ibid.

15. Jean-André De Luc, « $12^{\mathrm{e}}$ lettre à M. Delamétherie », Observations sur la Physique, $\mathrm{n}^{\circ} 38,1791$, p. 101.

16. Cuvier, « Mémoire sur les espèces d'éléphants vivantes et fossiles », op. cit., p. 12.

17. Jacques Christophe Valmont de Bomare, Dictionnaire raisonné universel d'histoire naturelle, Paris, Brunet, $2^{\mathrm{e}}$ édition, 1775, t. 2, p. 750.

18. Johann Friedrich Blumenbach, Beyträge zur Naturgeschichte, Göttingen, Johann Christian Dieterich, 1790, p. 119.

19. Johann Friedrich Blumenbach, Abbildungen naturhistorischer Gegenstände, Göttingen, J. C. Dieterich, 1797, t. II, p. 23.

20. Cédric Grimoult, Histoire des théories scientifiques de l'extinction des espèces, Paris, Ellipses, 2014, p. 32-55.

21. Goulven Laurent, Paléontologie et évolution en France de 1800 à 1860. Une histoire des idées de Cuvier et Lamarck à Darwin, Paris, Éd. du CTHS, 1987, p. 35-36.

22. Maurice Champion, La Fin du monde et les comètes au point de vue historique et anecdotique, Paris, A. Delahays, 1859, p. 66-67 et 173-179.

23. Pierre-Simon de Laplace, Exposition du système du monde [1797], Paris, Bachelier, 1835, p. 234.

24. Cité par Thomas Medwin, Journal of the conversations of Lord Byron: Noted during a residence with his Lordship at Pisa, in the years 1821 and 1822, Londres, Henry Colburn, 1824, p. 282.

25. Cédric Grimoult, Évolutionnisme et fixisme en France: histoire d'un débat. 1800-1882, op. cit., p. 97-100.

26. Georges Wolfgang Knorr et Jean Ernest Emmanuel Walch, Recueil des monuments des catastrophes que le globe de la Terre a essuyées, Nuremberg, 1775, t. II, p. 129.

27. Ibid., p. 166.

28. Pierre Simon Pallas, Observations sur la formation des montagnes et les changements arrivés au globe, particulièrement à l'égard de l'empire russe, Saint-Pétersbourg, Imprimerie impériale des sciences, 1777, p. 38.

29. Déodat de Gratet de Dolomieu, « Mémoire sur les pierres composées et les roches ", Journal de Physique, de Chimie, d'Histoire Naturelle et des Arts, 1791, nov., 39, partie II, p. 384, 389-391, 398-400, 403-404.

30. Ibid., p. 495.

31. Cuvier, Recherches sur les ossements fossiles de quadrupèdes, Paris, Deterville, 1812, t. I, p. 38.

32. Ibid., p. 24.

33. Lamarck, Hydrogéologie, Paris, chez l'auteur, an X (1802), p. 75.

34. Lamarck, Système des animaux sans vertèbres, Paris, Deterville, an IX (1801), p. 24.

35. Cédric Grimoult, Le Développement de la paléontologie contemporaine, Genève-Paris, Droz, 2000, p. 20.

36. Lamarck, Réfutation de la théorie pneumatique, Paris, Agasse, an IV (1795-1796), p. 447.

37. Lamarck, Philosophie zoologique, Paris, Dentu et chez l'auteur, 1809, t. II, p. 187.

38. Lamarck, Système des animaux sans vertèbres, op. cit., p. 407.

39. Lamarck, « Coquillage », Nouveau Dictionnaire d'histoire naturelle, 1817, t. XVII, p. 553.

40. Lamarck, Hydrogéologie, op. cit., p. 69. 
41. Ibid., p. 73.

42. Ibid., p. 408-409.

43. Richard W. Burkhardt Jr., op. cit., p. 134.

44. Lamarck, "Suite des mémoires sur les fossiles des environs de Paris », Annales du Muséum d'Histoire Naturelle, 1803, t. 2, p. 223.

45. Lamarck, Hydrogéologie, op. cit., p. 411.

46. Cuvier, « Extrait d'un ouvrage sur les espèces de quadrupèdes dont on a trouvé les ossements dans l'intérieur de la terre, adressé aux savants et amateurs des sciences, adressé aux savants et aux amateurs des sciences ", Journal de Physique, 1801, 52, p. 260.

47. Cédric Grimoult, Histoire des théories scientifiques de l'extinction des espèces, op. cit., p. 74-81.

48. Charles Lyell, Principes de géologie, ou illustrations de cette science, empruntées aux changements modernes que la Terre et ses habitants ont subis, Paris, Langlois et Leclercq, t. 1, 1843 (éd. orig. 1830). Trad : Tullia Meulien, p. 337.

49. Cuvier, Recherches sur les ossements fossiles de quadrupèdes, op. cit., t. 1, p. 74.

50. Cédric Grimoult, Histoire des théories scientifiques de l'extinction des espèces, op. cit., p. 74-75.

51. Lamarck, Philosophie zoologique, op. cit., t. I, p. 236.

52. Lamarck, Système des animaux sans vertèbres, op. cit., p. 13.

53. Denis Diderot, Éléments de physiologie, 1774, in: Euvres complètes, éd. par Jules Assézat et Maurice Tourneux, Paris, Garnier frères, 1875-1876, t. 1, p. 336

54. Charles-Georges Leroy [anonyme], Lettres sur les animaux, Paris, Saugrain jeune, 1781, p. 287.

55. Georges Cabanis, Rapports du physique et du moral de l'homme, Paris, Crapart, Caille et Ravier, an X-1802, t. II, p. 253.

56. Cédric Grimoult, La preuve par neuf. Les révolutions de la pensée évolutionniste, Paris, Ellipses, 2009, p. 154-161.

57. Lamarck, Recherches sur l'organisation des corps vivants, Paris, Maillard, an X (1802), p. 43.

58. Ibid., p. 42.

59. Lamarck, Philosophie zoologique, op. cit., t. II, p. 463.

60. Martin J. S. Rudwick, op. cit., p. 507, en note.

61. Cuvier, Recherches sur les ossements fossiles de quadrupèdes, op. cit., t. I, p. 11.

62. Ibid., p. 11-12.

63. Lamarck, Hydrogéologie, op. cit., p. 12.

64. Ibid., p. 38.

65. Ibid., p. 17.

66. Cuvier, Recherches sur les ossements fossiles de quadrupèdes, op. cit., t. I, p. 11, en note.

67. Ibid.

68. John Fleming, The Philosophy of zoology: or a general view of the structure, functions, and classification of animals, Edinburgh, Archibald Constable \& Co., 1822, t. II, p. 12-13.

69. Gabriel Gohau, Les Sciences de la Terre aux XVII et XVIII ${ }^{e}$ siècles. Naissance de la géologie, Paris, A. Michel, 1990, p. 286.

70. Cuvier, Recherches sur les ossements fossiles de quadrupèdes, op. cit., t. I, p. 74.

71. Cf. Cédric Grimoult, Lamarck, Paris, Ellipses, 2020, à paraître.

72. Cédric Grimoult, «Le débat sur le ponctualisme en France : le colloque de Dijon en 1982 », Revue d'histoire des sciences, t. 62-2, juillet-décembre 2009, p. 423-454. 


\section{RÉSUMÉS}

Dans le contexte de la Révolution française, Lamarck affronte Cuvier au sujet de l'existence, sur la Terre du passé, de catastrophes soudaines, responsables de l'extinction des formes fossiles et empêchant leurs transformations successives pouvant expliquer l'origine des espèces actuelles. Leurs articles se répondent explicitement et présentent des arguments majeurs, ainsi qu'une surprenante simplification de leurs démonstrations. Cet article aborde la question des causes, des modalités et des enjeux de ce combat majeur dans l'histoire des idées, dont les échos se font encore sentir dans l'actualité des sciences de la vie et de la Terre.

In the context of the French Revolution, Lamarck fights Cuvier about the existence, upon the Earth of the past, of sudden catastrophes, which could be responsible of the extinction of the fossil species and prevent their transformations into the actual living forms. Their papers hold a dialogue, present some key arguments and also a quite astonishing simplification of their demonstrations. This article tackles the question of the causes, the modes and the main issues of this major struggle of ideas, which echoes can still be heard in the life and Earth sciences today.

\section{INDEX}

Mots-clés : évolution, géologie, révolution, catastrophisme, fossile

Keywords : evolution, géology, revolution, catastrophism, fossils

\section{AUTEUR}

\section{CÉDRIC GRIMOULT}

Centre d'Histoire Culturelle des Sociétés Contemporaines, Université Versailles-Saint-Quentin 